\title{
X-ray scatter measurements from thermally slumped thin glass substrates for the HEFT hard x-ray telescopes
}

\begin{abstract}
Ahsen M. Hussain, Finn Erland Christensen, Mario A. Jimenez-Garate, William W. Craig, Charles J. Hailey, et al.
\end{abstract}

Ahsen M. Hussain, Finn Erland Christensen, Mario A. Jimenez-Garate, William W. Craig, Charles J. Hailey, Todd R. Decker, Marcela Stern, David L. Windt, Peter H. Mao, Fiona A. Harrison, Giovanni Pareschi, Manuel Sanchez del Rio, Alexei Souvorov, Andreas K. Freund, Remi Tucoulou, Anders Madsen, Christian B. Mammen, "X-ray scatter measurements from thermally slumped thin glass substrates for the HEFT hard x-ray telescopes," Proc. SPIE 3766, X-Ray Optics, Instruments, and Missions II, (29 September 1999); doi: 10.1117/12.363635 Instrumentation, 1999, Denver, CO, United States 


\title{
X-ray Scatter Measurements from Thermally Slumped Thin Glass Substrates for the HEFT Hard X-ray Telescopes
}

\author{
Ahsen M. Hussain ${ }^{a}$, Finn E. Christensen ${ }^{a}$, Mario A. Jimenez-Garate $^{b}$, \\ William W. Craig ${ }^{b}$, Charles J. Hailey ${ }^{b}$, Todd R. Decker ${ }^{b}$, Marcela Stern ${ }^{b}$ \\ David L. Windt ${ }^{c}$, Peter H. Mao ${ }^{d}$, Fiona A. Harrison ${ }^{d}$, \\ Giovanni Pareschi ${ }^{e}$, Manuel Sanchez del Rio ${ }^{f}$, Alexei Souvorov $f$, \\ Andreas K. Freund ${ }^{f}$, Remi Tucoulou ${ }^{f}$, Anders Madsen ${ }^{g} \&$ Christian Mammen $^{g}$ \\ ${ }^{a}$ Danish Space Research Institute, Juliane Maries Vej 30, \\ Copenhagen Dk-2100, Denmark. \\ ${ }^{b}$ Columbia Astrophysics Laboratory, Columbia University, New York, USA. \\ ${ }^{c}$ Bell Laboratories, Lucent Technologies, New Jersey, USA. \\ ${ }^{d}$ Space Radiation Laboratory, California Institute of Technology, Pasadena, California, USA. \\ ${ }^{e}$ Osservatorio Astronomico di Brera-Milano, Merate, Italy.

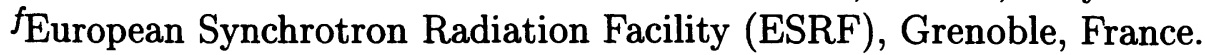 \\ ${ }^{g}$ Ørsted Laboratory, University of Copenhagen, Copenhagen, Denmark.
}

\begin{abstract}
We have performed X-ray specular reflectivity and scattering measurements of thermally slumped glass substrates on X-ray diffractometers utilizing a rotating anode X-ray source (8.048 keV) at the Danish Space Research Institute (DSRI) and synchrotron radiation (18 \& $28 \mathrm{keV}$ ) at the European Synchrotron Radiation Facility (ESRF) optics Bending Magnet (BM05) beamline. In addition, we tested depth graded W/Si multilayer-coated slumped glass using $\mathrm{X}$-ray specular reflectivity measurements at $8.048 \mathrm{keV}$ (DSRI) \& $28 \mathrm{keV}$ (ESRF) and energy-dispersive measurements in the $20-50 \mathrm{keV}$ range at a double-axis diffractometer at the Ørsted Laboratory, University of Copenhagen. The thermally slumped glass substrates will be used to fabricate the hard X-ray grazing incidence optics for the $H i g h$ Energy Focusing Telescope (HEFT). ${ }^{7,8}$ We compared the measurements to the SODART-mirrors ${ }^{1}$ from the $S R G^{17}$ telescope mission program. The surface scatter measurements of the thermally slumped glass substrates yields Half Power Diameters (HPD's) of single-bounce mirrors of full-illuminated lengths of $\sim 40$ arcseconds for typical substrates and as low as $\sim 10$ arcseconds for the best substrates, whereas the SODART mirrors yields HPD's of $\sim 80$ arcseconds with very little variation. Both free-standing glass substrates and prototype mounted and multilayercoated optics were tested. The results demonstrate that the surface scatter contribution, plus any contribution from the mounting procedure, to the Half Power Diameter (HPD) from a telescope using the slumped glass optics will be in the subarcminute range. In addition we measured low surface microroughness, yielding high reflectivity, from the glass substrates $(\sim 2 \AA)$, as well as from the depth graded W/Si multilayer-coated glass (interfacial width $\sim 4.2 \AA$ ).
\end{abstract}

Keywords: X-ray Reflectivity, X-ray Scattering, Synchrotron Radiation, Hard X-ray Telescopes, X-ray Optics, Segmented Optics, Wolter-I Optics, Thermal Slumping, Depth Graded Multilayers.

Correspondence: Ahsen M. Hussain:

E-mail: ahsen@dsri.dk, Tel: (+45) 35325735 , Fax: (+45) 35362475 


\section{INTRODUCTION}

The $H E F T^{7,8}$ telescope consists of an $1.25 \mathrm{~m}$ (diameter) array of 14 co-aligned conical approximation Wolter-I mirror modules, each focusing hard X-rays/soft gamma-rays (20-100 keV) onto individual solid-state Cadmium Zinc Telluride $(\mathrm{CdZnTe})$ pixel detectors positioned at a focal length of $6 \mathrm{~m}$. Each module consists of 72 nested shells (consisting of a front and back section, approximating the hyperboloid and paraboloid sections of a Wolter-I reflector), produced in quadrant segments. A total of 8064 quadrant segments is then required for the entire telescope array. The shells are $20 \mathrm{~cm}$ long in the direction of the optical axis and vary in radius from $4 \mathrm{~cm}$ to $12 \mathrm{~cm}$.

The large numbers of segments required for the telescope demands substrate structures that can be easily fabricated at low cost. In addition the substrate must be of high strength and must be able to maintain its figure.

Columbia University, the Danish Space Research Institute (DSRI) and California Institute of Technology have worked in a joint effort on development and characterization of new candidate optics for future Hard X-ray telescopes, in particular for the High-Energy Focusing Telescope $(H E F T)^{7,8}$ and the Constellation- $X^{5,6,18}$ NASA missions.

The optic chosen for the HEFT telescope is the thin $(0.3 \mathrm{~mm}$ ) DESAG AF45 (a Schott Group Product) glass sheet. The DESAG (AF45) boro-silicate glass firepolished microsheets are originally developed for flat panel displays (by means of a process called "diffusion overflow", where any mechanical contact with the glass surface is avoided in the critical glass production process ${ }^{13}$ ), and are thus inherently smooth and flat. ${ }^{11}$ The glass is slumped under gravity in an oven at temperatures around $700-750^{\circ}$ (thus below the softening point of the glass and above the annealing point) into the required shape, determined by a cylindrical quartz tube mandrel, cut in half along its length.

In order to achieve a broad-band energy response in the hard X-ray band up to $100 \mathrm{keV}$, all of the individual segments in the HEFT telescope will be coated with depth graded multilayers. ${ }^{2}$ A study has been conducted to optimize the coating of the mission. ${ }^{14}$ The present optimized design utilizes W/Si graded d-spacing multilayers on the outer shells in the 20-69.5 keV energy band (W having its K-absorption edge at $69.5 \mathrm{keV}$ ). Several different graded multilayer coatings are currently being investigated, to be utilized on the inner shells for the $E>69.5 \mathrm{keV}$ energy range.

The thermally slumped glass substrates will be coated both at the new DC magnetron sputtering Multilayer Deposition System (MDS) ${ }^{10}$ at DSRI and at a DC magnetron sputter deposition system at Bell Laboratories (Lucent Technologies). ${ }^{20}$ The multilayers tested in this presentation were coated at the latter facility.

The following sections present the experimental setups and the results, followed by the conclusions.

\section{EXPERIMENTAL SETUPS}

We performed X-ray characterization measurements at several different experimental setups :

1. $8.048 \mathrm{keV}$ Specular Reflectivity Measurements: The $8.048 \mathrm{keV}$ specular reflectivity measurements were made at a double-axis diffractometer at DSRI. ${ }^{3}$ The system utilizes a $12 \mathrm{~kW}$ Rigaku rotating anode $\mathrm{X}$-ray source (working at $\mathrm{Cu}-\mathrm{K}_{\alpha_{1}}(8.048 \mathrm{keV})$ ). The beam is confined by two slits $(1.0 \mathrm{~mm}$ (width) $\times$ $2.0 \mathrm{~mm}$ (height)) in front of the asymmetric cut $\mathrm{Ge}(111)$ monochromator and beam compressor. An additional guard slit $(0.1 \mathrm{~mm} \times 2.0 \mathrm{~mm})$ is placed in front of the sampleholder. The resolution of the setup is $\sim 1$ arcminute FWHM. The setup utilizes a one-dimensional position sensitive detector for simultaneous measurements of the specular reflectivity and scattering. This combined with subsequent analysis software allows for detecting scattering from roughness on lengthscales up to $25 \mu \mathrm{m}$. The rootmean-square (r.m.s) roughness derived from the specular reflectivity data is thus the r.m.s roughness averaged over all lengthscales up to $25 \mu \mathrm{m}$.

2. $8.048 \mathrm{keV}$ Scatter Measurements: For the $8.048 \mathrm{keV}$ high-resolution ( $\sim 5$ arcsecond FWHM) scatter measurements we changed the setup to a triple-axis diffractometer configuration, utilizing high-resolution perfect channel-cut monochromator and analyzer crystals (both $\mathrm{Si}(220)$ ) in a non-dispersive configuration. 
The high resolution of this setup allows for detection of true scattering from lengthscales up to $\sim 1800 \mu \mathrm{m}$ at a typical grazing incidence angle of 0.2 degrees, as well as detection of $\sim 5$ arcsecond or larger figure errors. We performed the scattering measurements by fixing the incidence angle, $\theta_{i}$, on the sample and subsequently rotating the analyzer crystal to probe the scattered radiation.

3.18 \& $28 \mathrm{keV}$ Reflectivity and Scatter Measurements: We made the hard X-ray, $18 \mathrm{keV} \& 28$ $\mathrm{keV}$, specular reflectivity and scatter measurements on a double-axis diffractometer at the optics Bending Magnet beamline (BM05) at the European Synchrotron Radiation Facility (ESRF) in Grenoble (France), with an angular divergence of the incoming beam of $\sim 1.03$ arcsecond FWHM for the specular reflectivity measurements. Given the width of the detector slit used in the specular reflectivity measurements, the data allowed for detection of r.m.s roughness on lengthscales up to around $1 \mu \mathrm{m}$. For the $18 \mathrm{keV}$ and 28 $\mathrm{keV}$ scatter measurements, the detector slit was varied for coarse and fine scatter measurements, yielding resolutions of $\sim 17$ arcseconds and $\sim 3$ arcseconds FWHM, respectively. Thus detecting scattering/figure errors on the same lengthscale range/angular range as the $8.048 \mathrm{keV}$ scatter measurements.

4. 20-50 keV Energy Dispersive Measurements: For the energy-dispersive measurements, we used a collimated "white beam" (bremsstrahlung spectrum) from an $18 \mathrm{~kW}$ Rigaku rotating anode on a doubleaxis diffractometer at Ørsted Laboratory (University of Copenhagen), yielding X-ray photons in the $\sim 2-60$ $\mathrm{keV}$ energy range. The setup utilizes a $\mathrm{W}$ filament and a $\mathrm{Cu}$ anode. The usable range for measurements is above approximately $20 \mathrm{keV}$, avoiding any characteristic spectral lines of $\mathrm{W}$ and $\mathrm{Cu}$. The upper limit was chosen as $\sim 50 \mathrm{keV}$. The detector used in these measurements was a Nitrogen-cooled Ge solid state detector in conjuction with a multichannel analyzer, having a resolution of $1-3 \%$ over the $20-50 \mathrm{keV}$ energy range. The measurements were performed by rotating the sample to a fixed grazing angle $\theta$ and collect the reflected intensity (at $2 \theta$ ) as a function of the energy of the reflected photons.

\section{RESULTS \& DISCUSSION}

We analyzed the reflectivity data using the IMD software package, ${ }^{19}$ which applies an algorithm based on Parrat's recursive application ${ }^{16}$ of the Fresnel equations, using the Névot-Croce factor ${ }^{15}$ for the roughness. The optical constants used in IMD for the different materials are taken from the updated tables at Center for X-ray Optics (CXRO) at Lawrence Berkeley National Laboratory (LBNL) and tables at Lawrence Livermore National Laboratory (LLNL). In the case of scattering, the main quantitative number extracted from the data was the the Half Power Diameter (HPD) extracted from encircled energy calculations.

\subsection{Free-standing Thermally Slumped Glass Substrates}

In order to have a better comparison of the non-coated free-standing slumped DESAG glass with the SODARTmirrors (gold-coated dip-lacquered rolled aluminum foils), the glass substrates were coated with gold (approximately 300-400 $\AA$ ), using a sputter deposition system (at "Koral Labs" (Minnesota, USA)).

\subsubsection{Reflectivity Data}

Figure 1 shows the reflectivity versus grazing incidence angle results obtained on a typical Au-coated slumped glass (diamonds) compared to the SODART-mirror (triangles) at $8.048 \mathrm{keV}$. The fits to the data indicate a surface microroughness, $\sigma$, of $8.0 \AA$ for the Au-surface on the glass and $2.3 \AA$ on the underlying glass-Au interface, whereas the SODART Au-surface has a microroughness of $10.5 \AA$ and $4.9 \AA$ on the underlying lacquer-Au interface (Carbon was used in the fit model as an approximation to the more complicated lacquer composition).

In a similar fashion, Figure 2 shows the comparison at $18 \mathrm{keV}$, with $\sigma$ 's of $7.4 \AA$ and $11.4 \AA$ for the Au-surface on the glass and the SODART-mirror, respectively, and $1.6 \AA$ and $5.6 \AA$ for the underlying interfaces, respectively. A smearing of the SODART-data as compared to the Au-coated slumped glass data is observed in Figure 2. This is caused by the fact that the SODART-mirror has significantly higher roughness on the lengthscales above $1 \mu \mathrm{m}$, leading to a scatter contribution in the specular reflectivity data, and thus an enhanced smearing of the data. 


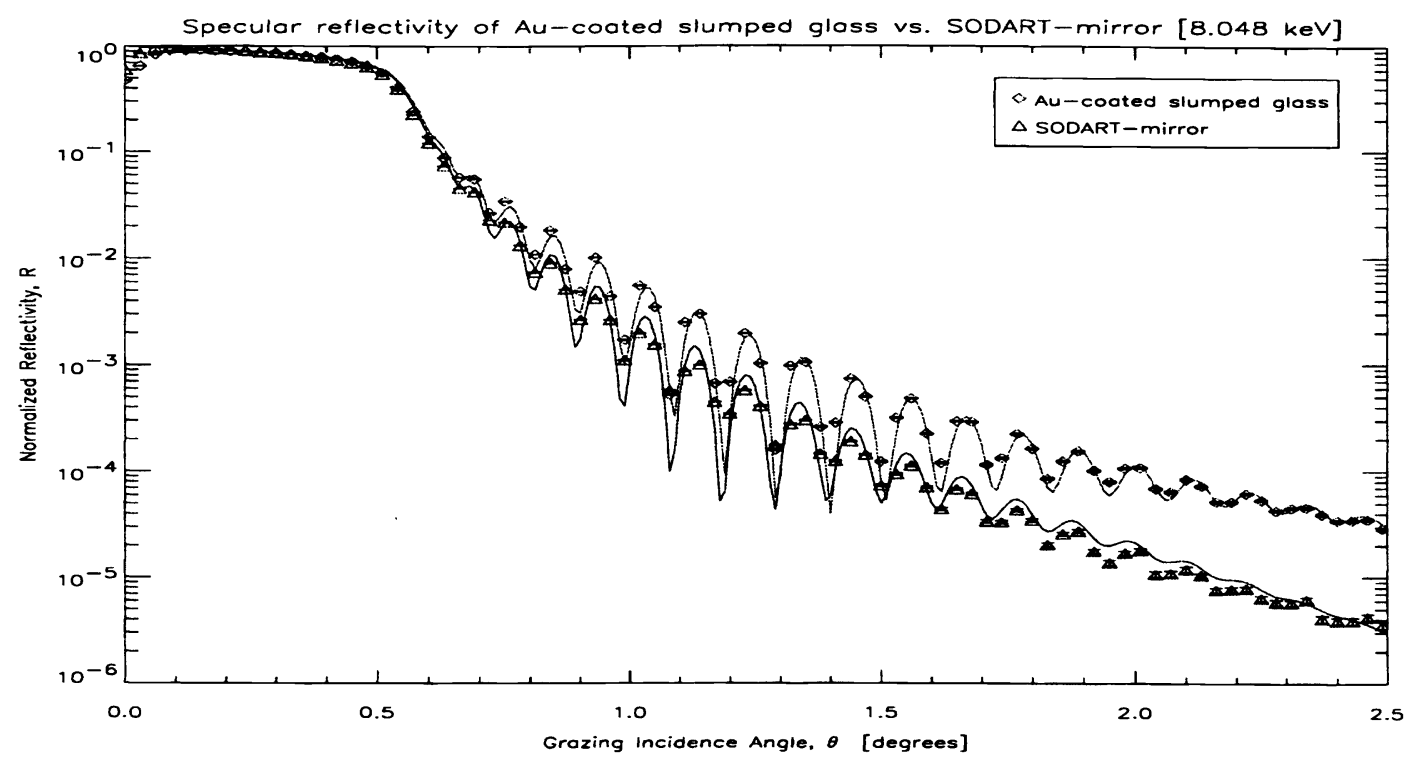

Figure 1. Specular reflectivity of a typical Au-coated slumped glass (diamonds) vs. SODART-mirrors (triangles) measured at $8.048 \mathrm{keV}$. The solid lines are the fits to the data. Note, the error-bars are smaller than the symbols.

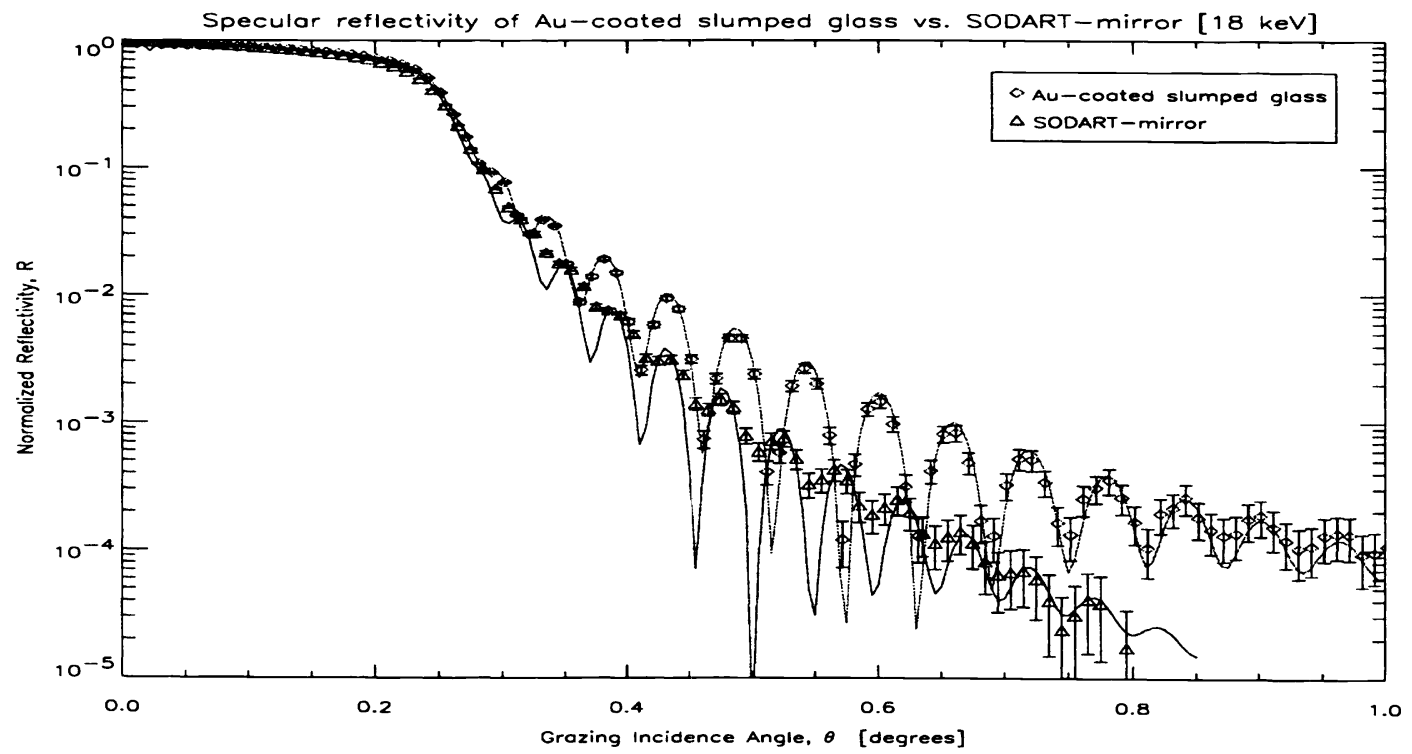

Figure 2. Specular reflectivity of typical Au-coated slumped glass (diamonds) vs. SODART-mirrors (triangles) measured at $18 \mathrm{keV}$. The solid lines are the fits to the data. 


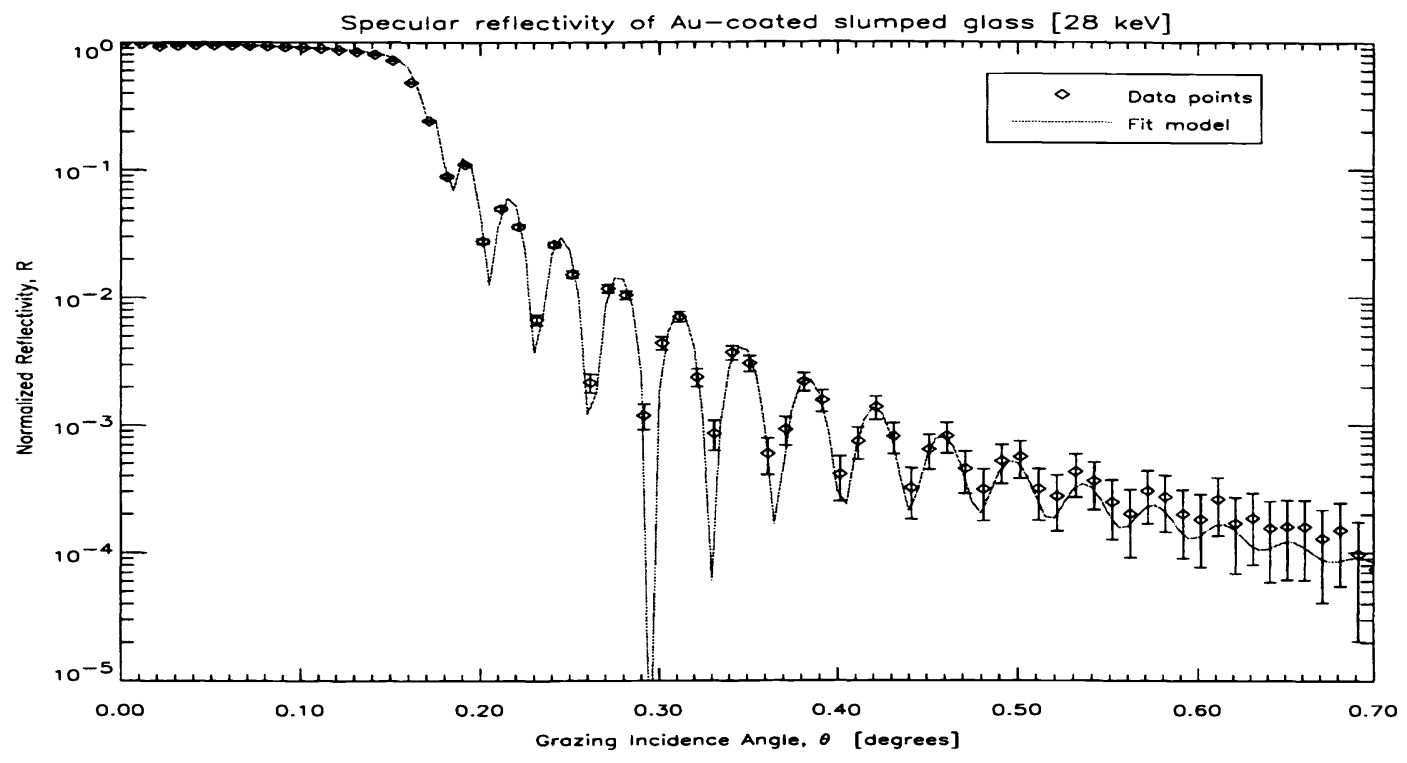

Figure 3. Specular reflectivity of a typical Au-coated slumped glass (diamonds) at $28 \mathrm{keV}$. The solid line is the fit to the data.

A detailed model of the correlation length of the roughness is required to separate the scatter from the specular reflectivity in the data analysis. At present such a detailed model is not available. A detailed model will however only change the quality of the fit, and not the derived roughness parameters.

We tested the Au-coated slumped glass at $28 \mathrm{keV}$ (see Figure 3), and the surface roughness results obtained are similar to the $18 \mathrm{keV}$ case, i.e. $8.0 \AA$ and $1.6 \AA$ on the Au-surface and underlying interface, respectively.

The roughnesses obtained are quite consistent given the change of lengthscales probed at $8.048 \mathrm{keV}$ and at the harder X-ray energies. The fact that the gold coating on the SODART-mirror was done by thermal evaporation as opposed to sputtering explains the higher Au-surface roughness on the SODART-mirror.

The lacquer surface on the SODART-mirror has substantially higher roughness than the glass-Au interface on the slumped glass on the lengthscales probed in these measurements (up to $25 \mu \mathrm{m}$ at the $8.048 \mathrm{keV}$ measurements and up to around $1 \mu \mathrm{m}$ at $18 \mathrm{keV}$ and $28 \mathrm{keV}$ ). We measure roughness as low as $1.6 \AA$ on the glass-Au interface on the slumped glass, consistent with the promising results obtained from coating multilayers directly on the glass (see section 3.3).

\subsubsection{Scattering Data}

We performed the scattering measurements at grazing incidence angles of $0.1,0.2$, and 0.4 degrees at $8.048 \mathrm{keV}$, and 0.15 degrees at $18 \mathrm{keV}$ and $28 \mathrm{keV}$. In addition, the guard-slit opening was varied in the different setups as well, thus changing the illuminated surface length during the experiments, ranging from a few millimeter to the full size of the substrates $(\sim 20 \mathrm{~cm})$.

The high resolution scatter data from the best-case free-standing Au-coated slumped glass measured at $8.048 \mathrm{keV}$, with a grazing incidence angle, $\theta_{i}$, of 0.2 degrees is shown in Figure 4, with the intensity (diamonds) shown as a function of the analyzer offset (in arcseconds). The direct beam (resolution function) of the setup (triangles) 


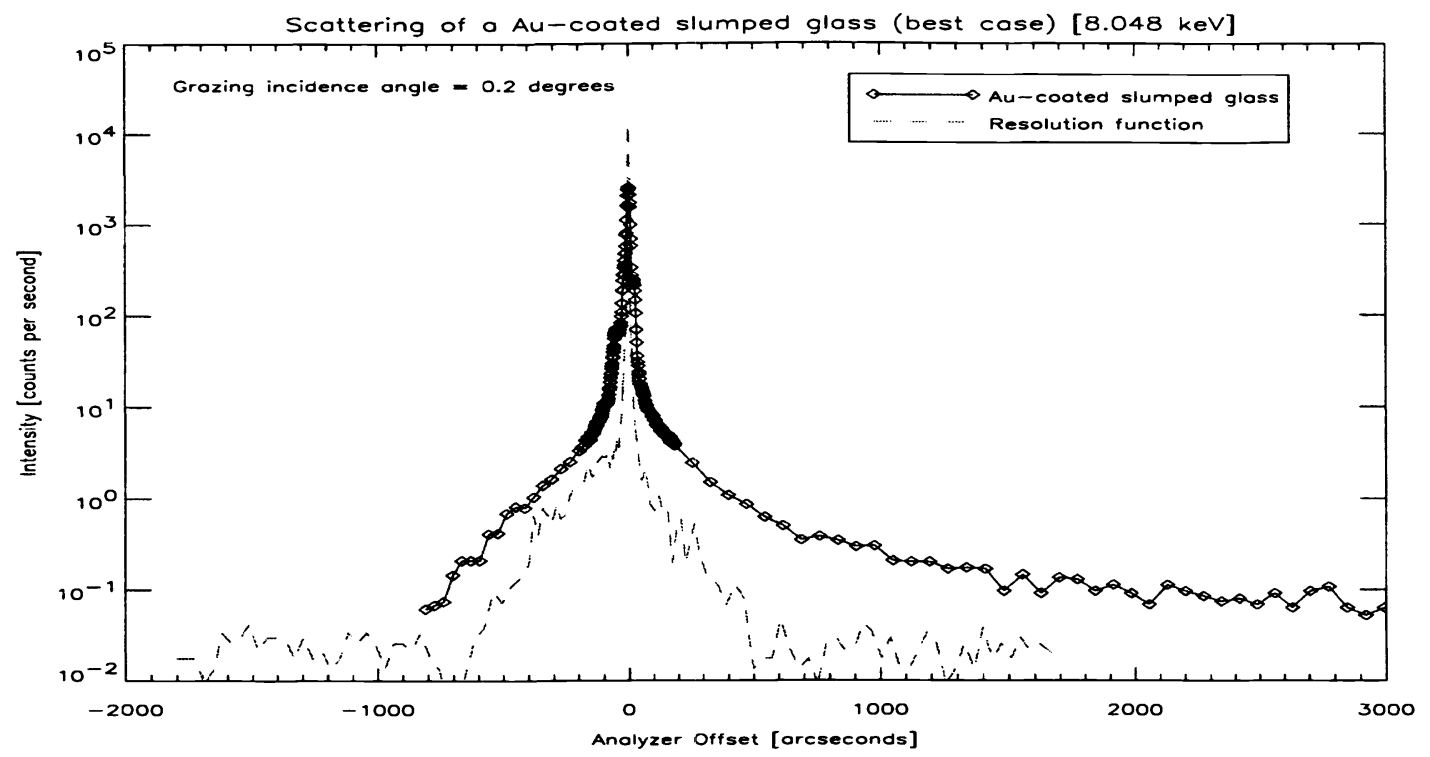

Figure 4. Scattering profile of a free-standing Au-coated slumped glass (best-case) measured at $8.048 \mathrm{keV}$. The connected diamonds represent the data, and the dashed line is the direct beam resolution function.

is likewise shown, correctly normalized, in the plot. The illuminated length of the substrate is around $15 \mathrm{~cm}\left(\frac{3}{4}\right.$ of a foil length) in this case. Figure 5 shows the corresponding encircled energy (diamonds) as a function of the angular diameter around the center of the scattering profile shown in Figure 4. We measure a Half Power Diameter (HPD) of 9.9 arcseconds. For typical substrates HPD's of around 40 arcseconds are obtained for full $20 \mathrm{~cm}$ length illumination. An encircled energy curve for such a substrate is shown (triangles) in Figure 5 as well, with a HPD of 44.6 arcseconds.

The Half Power Diameters (HPD's) obtained from the scatter data of a typical Au-coated slumped glass substrate measured at $8.048 \mathrm{keV}$ (diamonds), $18 \mathrm{keV}$ (triangles) and $28 \mathrm{keV}$ (squares) are shown in Figure 6, as a function of the illuminated surface length of the substrates.

The same procedure was also followed for the SODART-mirror. The HPD's calculated for the SODART-mirror at $8.048 \mathrm{keV}$ (cross-signs) are also shown in Figure 6.

It is clearly seen that in the case of Au-coated slumped glass the HPD increases with the illuminated surface length, indicating that the scattering originates predominantly from large scale figure errors, and not from actual surface microroughness, in agreement with the small microroughness inferred from the specular reflectivity data. The SODART-mirror maintains a relatively constant HPD around 80 arcseconds for footprint sizes down to $5.0 \mathrm{~cm}$, indicating that the scattering originates on smaller lengthscales. A more detailed analysis of the scatter profile will be published elsewhere. ${ }^{9}$

The above results demonstrate that typical slumped glass substrates will allow for a two-bounce telescope with a scatter contribution (including figure errors) to the HPD of $\leq 1$ arcmin. We have also demonstrated that a more careful selection of the substrates will result in a significant reduction of this number. 


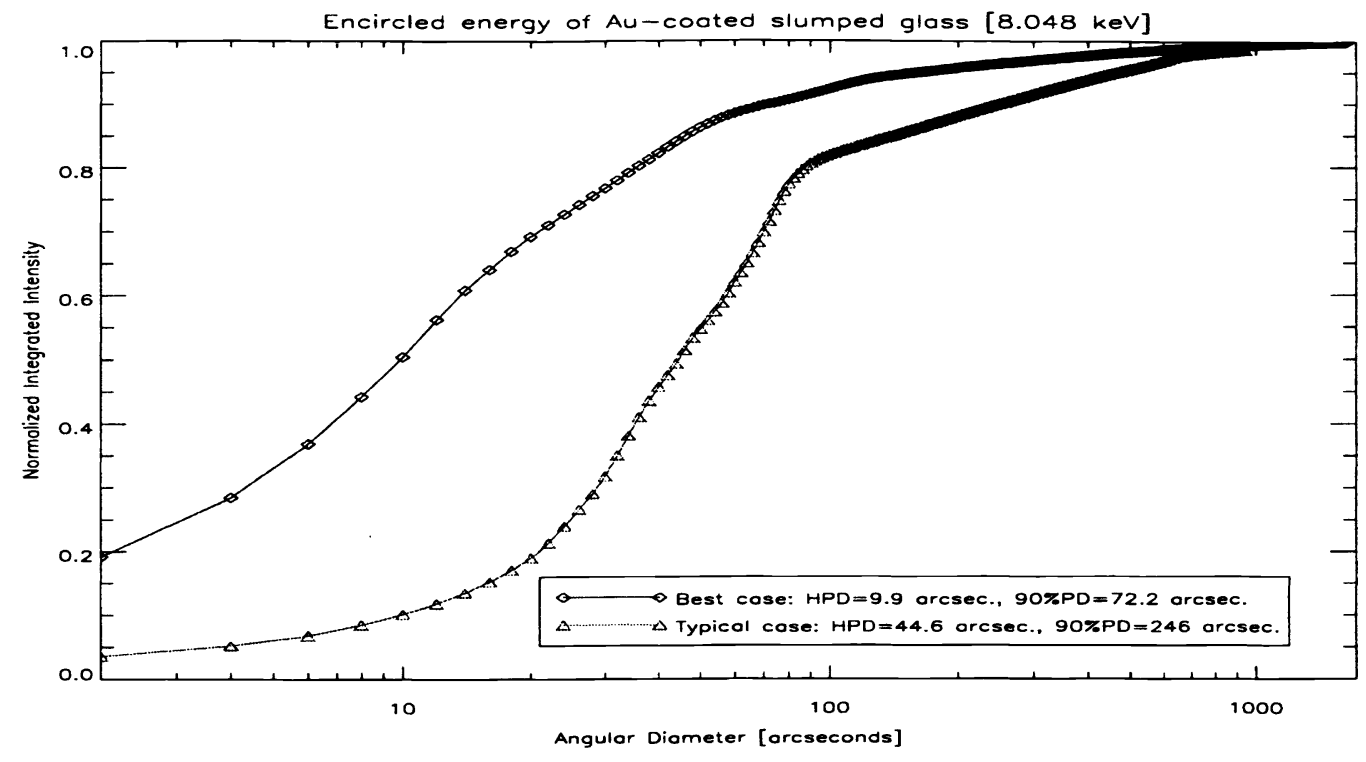

Figure 5. Encircled energy as a function of angular diameter around the center of the scattering profile for a Aucoated slumped glass measured at $8.048 \mathrm{keV}$. The connected diamonds represent the best case, whereas the connected triangles represent typical substrates.

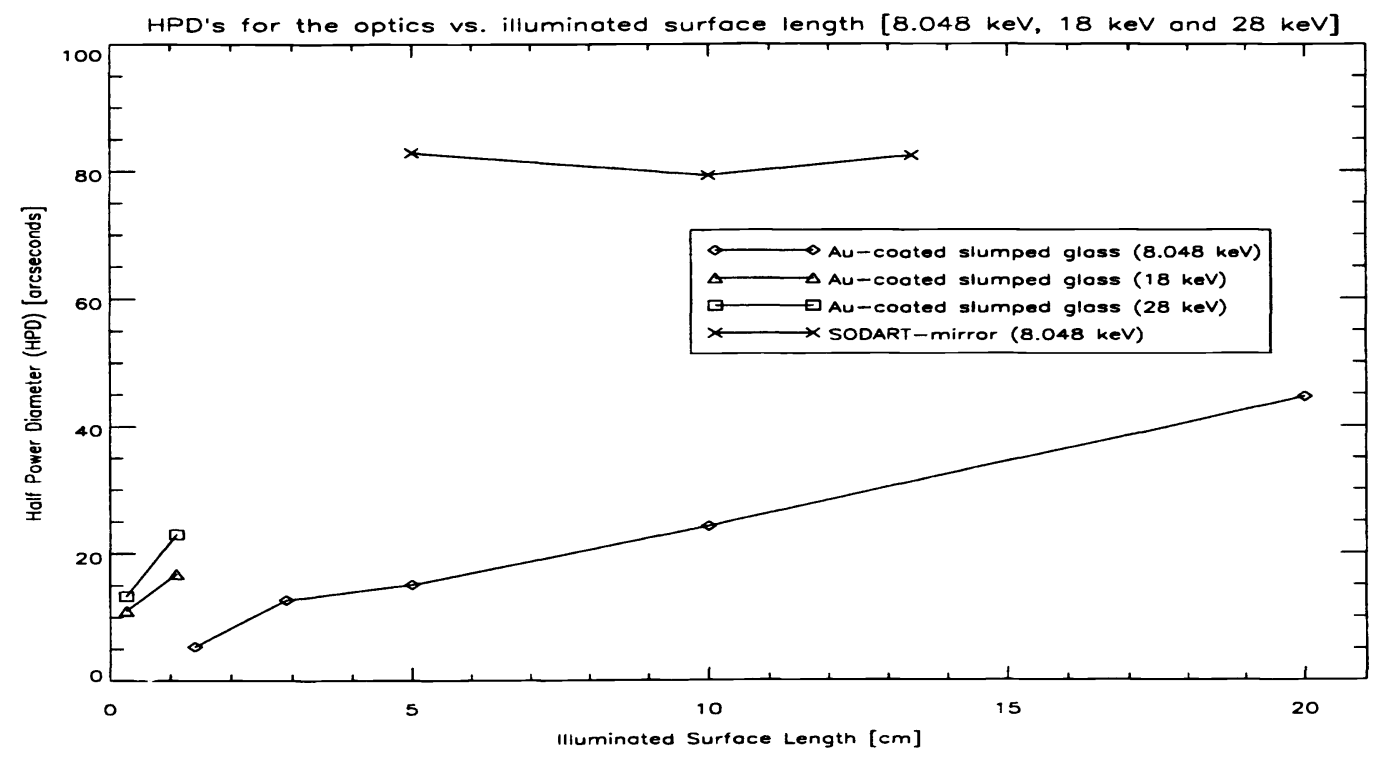

Figure 6. Half Power Diameter (HPD)'s as a function of the illuminated substrate surface length for the Au-coated slumped glass vs. SODART-mirrors. 


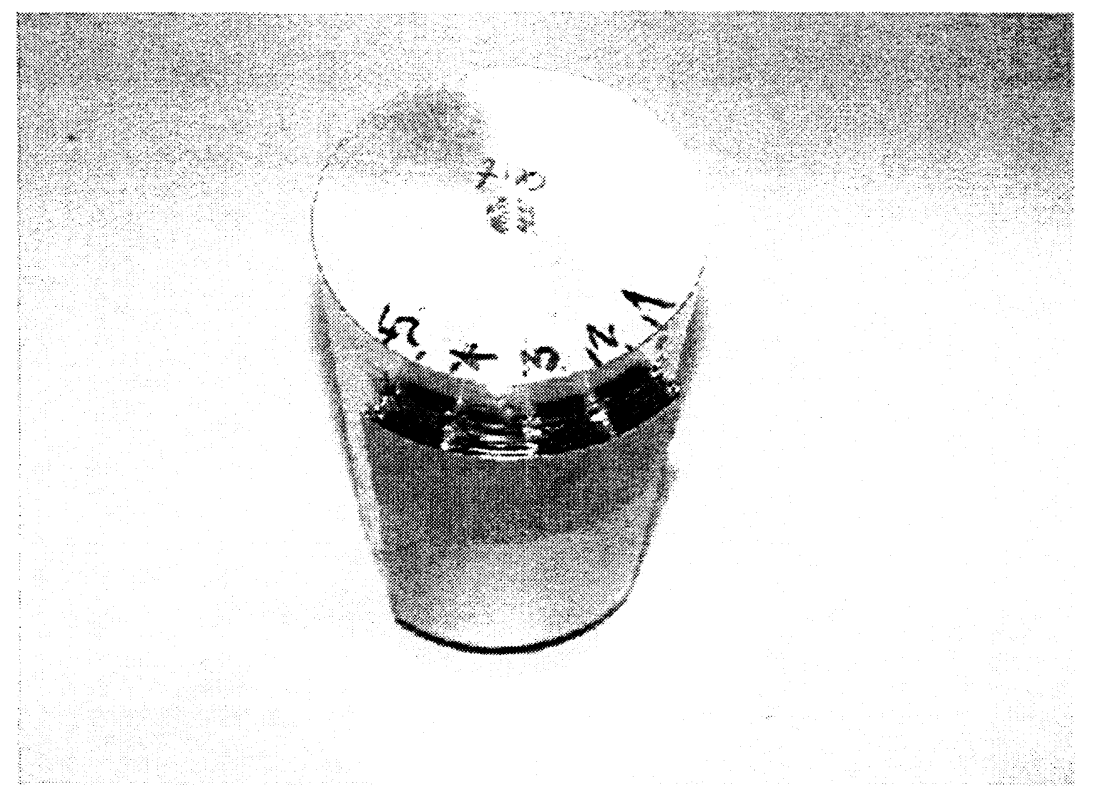

Figure 7. The HEFT 6-shell single bounce cylindrical prototype.

\subsection{Prototype Mounted Thermally Slumped Glass Substrates}

We built a prototype cylindrical fixture with 3 realistic W/Si multilayer-coated and 3 un-coated (no Au-layer) thermally slumped glass substrates mounted, using the scheme developed for HEFT. ${ }^{4}$ The cylinder consists of solid aluminum, with the foils carefully attached (see Figure 7 ). The different foils are separated by precisely machined graphite rods that position the substrates in the required cylindrical shape.

In order to compare the quality of a mounted optic with the free-standing optics, we performed a series of scattering measurements on the un-coated and multilayer-coated mounted foils. A representative scattering measurement of an un-coated mounted foil measured at 0.2 degree grazing incidence angle and the corresponding encircled energy curve are shown in Figure 8 and Figure 9, respectively.

Figure 9 shows that a HPD of around 40 arcsecond (in the range of a typical free-standing slumped glass substrate) is obtained from the prototype mounted slumped glass. In addition, no increase in HPD has been observed for the multilayer-coated foils. This clearly demonstrates that no additional scatter is introduced due to the multilayer coating or the mounting procedure developed for HEFT.

\subsection{Multilayer Coated Thermally Slumped Glass Substrates}

We coated free-standing slumped glass substrates with $\mathrm{W} / \mathrm{Si}$ depth graded multilayers at a sputter deposition facility at Bell Labs (Lucent Technologies) ${ }^{20}$ and subsequently tested the foils in X-ray specular reflectivity measurements both at $8.048 \mathrm{keV}$ and $28 \mathrm{keV}$. Figure 10 and 11 show the reflectivity results obtained on a $n=200$ bilayer W/Si depth graded multilayer. The grading of the bilayer thickness $d_{i}$ for the $i$ 'th bilayer (with the bilayer index $i$ ranging from 1 to $\mathrm{n}$, with $i=n$ being the bilayer next to the substrate) follows a power law distribution given by ${ }^{12}$

$$
d_{i}=\frac{a}{(b+i)^{c}}
$$




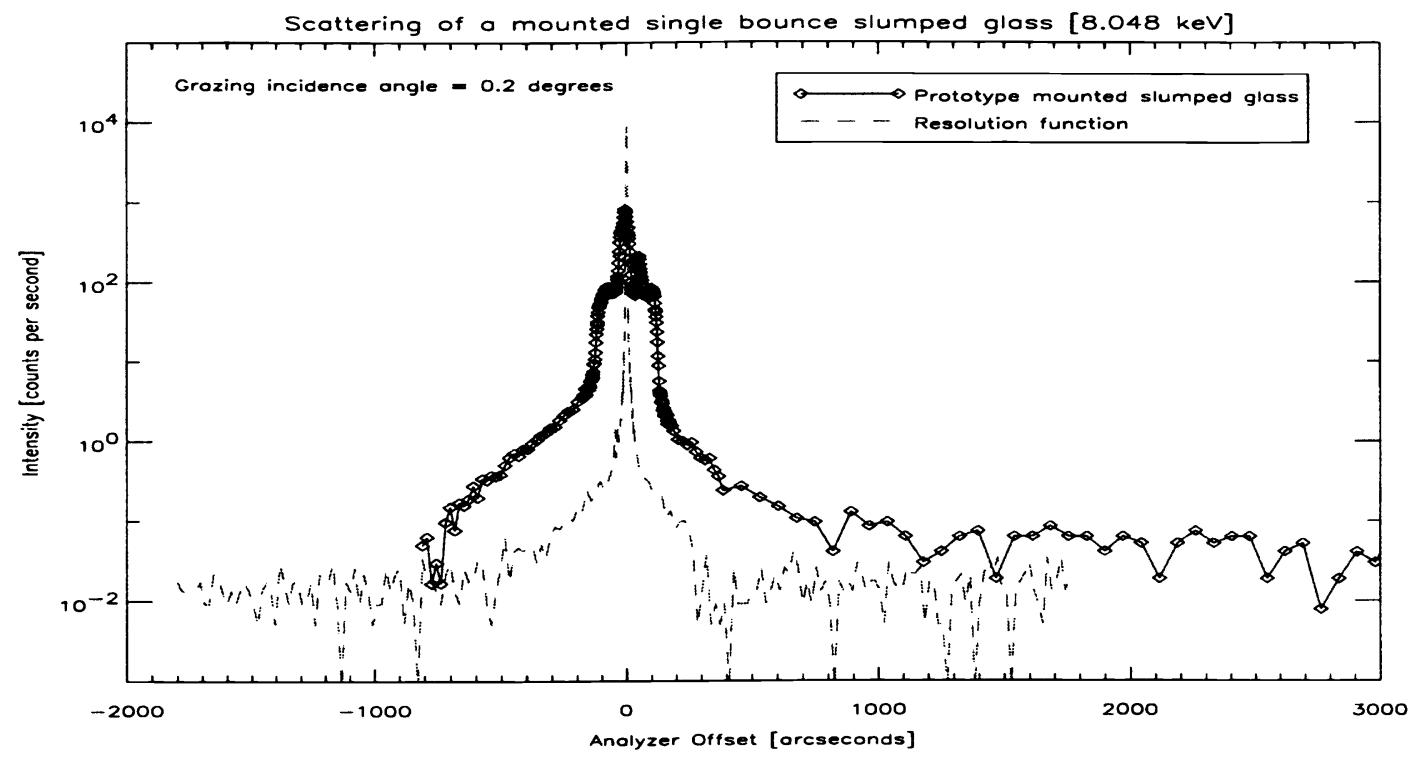

Figure 8. Scattering profile of a mounted single bounce thermally slumped glass optic measured at $8.048 \mathrm{keV}$. The connected diamonds represent the data, and the dashed line is the direct beam resolution function.

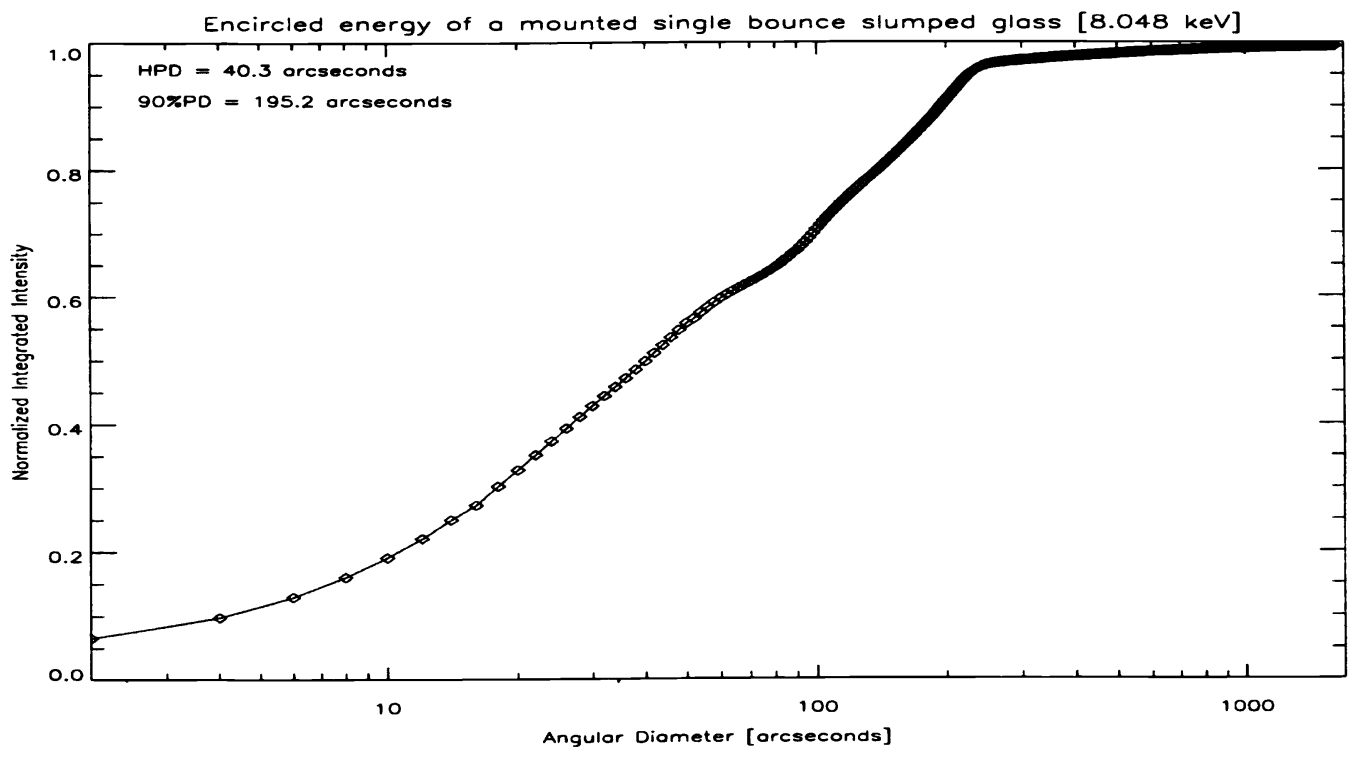

Figure 9. Encircled energy as a function of the angular diameter around the center of the scattering profile for a mounted thermally slumped glass measured at $8.048 \mathrm{keV}$. 


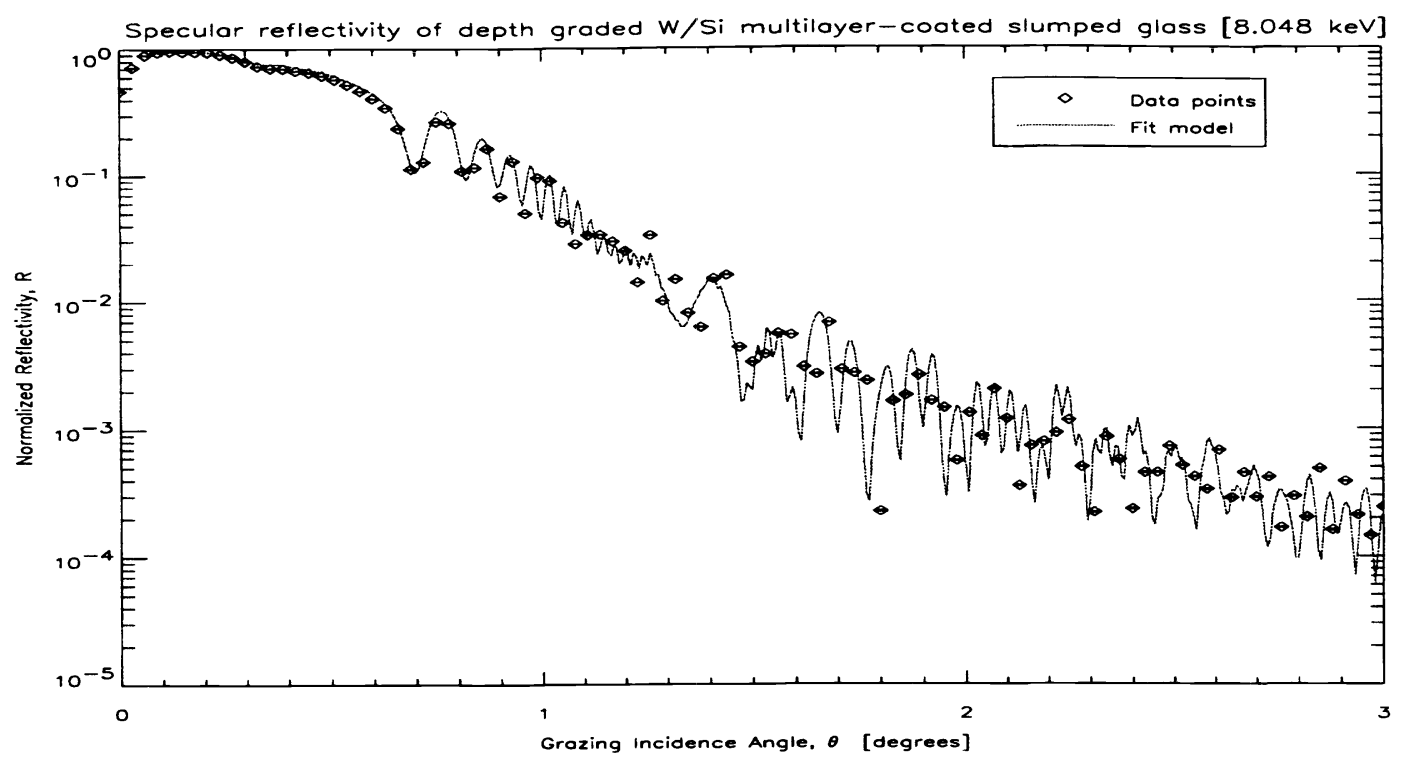

Figure 10. Specular reflectivity of $W / S i$ depth graded multilayer-coated slumped glass measured at $8.048 \mathrm{keV}$. The diamonds represents the data, and the solid line is the fit (see text for details). Note, the error bars are smaller than the symbols.

with the constants $a, b$ and $c$ nominally set to $93.45,-0.967$ and .227 , respectively. From these constants, nominal values of the minimum d-spacing, $d_{\min }$, and the maximum d-spacing, $d_{\max }$ can be determined.

In the fit shown in Figure 10 and Fig 11 the $\gamma$-value (the ratio of the thickness of the heavy layer (W) to the total bilayer thickness $d$ ) is 0.45 , and $d_{\min }$ and $d_{\max }$ are found to be $25.8 \AA$ and $197.8 \AA$, respectively, with the power law exponent $c$ set to the nominal value of 0.227 . The other parameters varied in the fit are the respective interfacial roughness parameters: 1 ) the glass substrate (approximated by $\mathrm{SiO}_{2}$ ) $-\mathrm{W}$ interfacial roughness $\sigma_{\text {sub }-W}$, 2 ) the "W-on-top-of-Si" interfacial roughness $\left.\sigma_{S i-W}, 3\right)$ the "Si-on-top-of-W" interfacial roughness $\left.\sigma_{W-S i}, 4\right)$ the top $\mathrm{Si}$ layer - oxidized $\mathrm{Si}$ layer interfacial roughness $\sigma_{\mathrm{Si}_{\mathrm{SiO}} \mathrm{S}_{2}}$ and finally 5) the $\mathrm{SiO}_{2}$ - vacuum interfacial roughness $\sigma_{\mathrm{SiO}_{2}-\text { vac }}$. The results obtained from the fits shown in Figure 10 and 11 are $2.3 \AA$ for $\sigma_{s u b-W}, 4.3 \AA$ for $\sigma_{S i-W}$, $4.1 \AA$ for $\sigma_{W-S i}, 5.0 \AA$ for $\sigma_{\mathrm{Si}_{-} \mathrm{SiO}_{2}}$ and finally $6.00 \AA$ for $\sigma_{\mathrm{SiO}_{2}-v a c}$. The interfacial roughness of the depth graded $\mathrm{W} / \mathrm{Si}$ multilayer is thus derived to be $\sim 4.2 \AA$ on lengthscales up to $25 \mu \mathrm{m}(8.048 \mathrm{keV})$ and at lengthscales up to around $1 \mu \mathrm{m}$ in the $28 \mathrm{keV}$ measurements.

A smearing of the measured data is observed in Figure 11 as compared to the data in Figure 10. This is caused by the fact that the size of the detector slit used in the $28 \mathrm{keV}$ measurement, does not allow for completely separating the specular reflectivity from the scattered intensity. Thus a scatter contribution is included in the specular data at $28 \mathrm{keV}$ as compared to the $8.048 \mathrm{keV}$ data, leading to the smearing of the data observed. A detailed model of the correlated roughness and the associated correlation length is required to separate the scatter from the specular reflectivity in the data analysis. At present such a detailed model is not available. A detailed model will however only change the quality of the fit, and not the derived roughness parameters.

The same free-standing $\mathrm{W} / \mathrm{Si}$ depth graded multilayer-coated substrate was also characterized at an energy-dispersive setup ( $\sim 20-50 \mathrm{keV})$ at the Ørsted Laboratory, University of Copenhagen, measuring the reflectivity as a function of photon energy, shown in Figure 12, at a grazing incidence angle of $0.2^{\circ}$, a realistic angle of reflection in the HEFT telescope. The diamonds are the data points and the solid line is the fit to the data. The fit model is identical with 


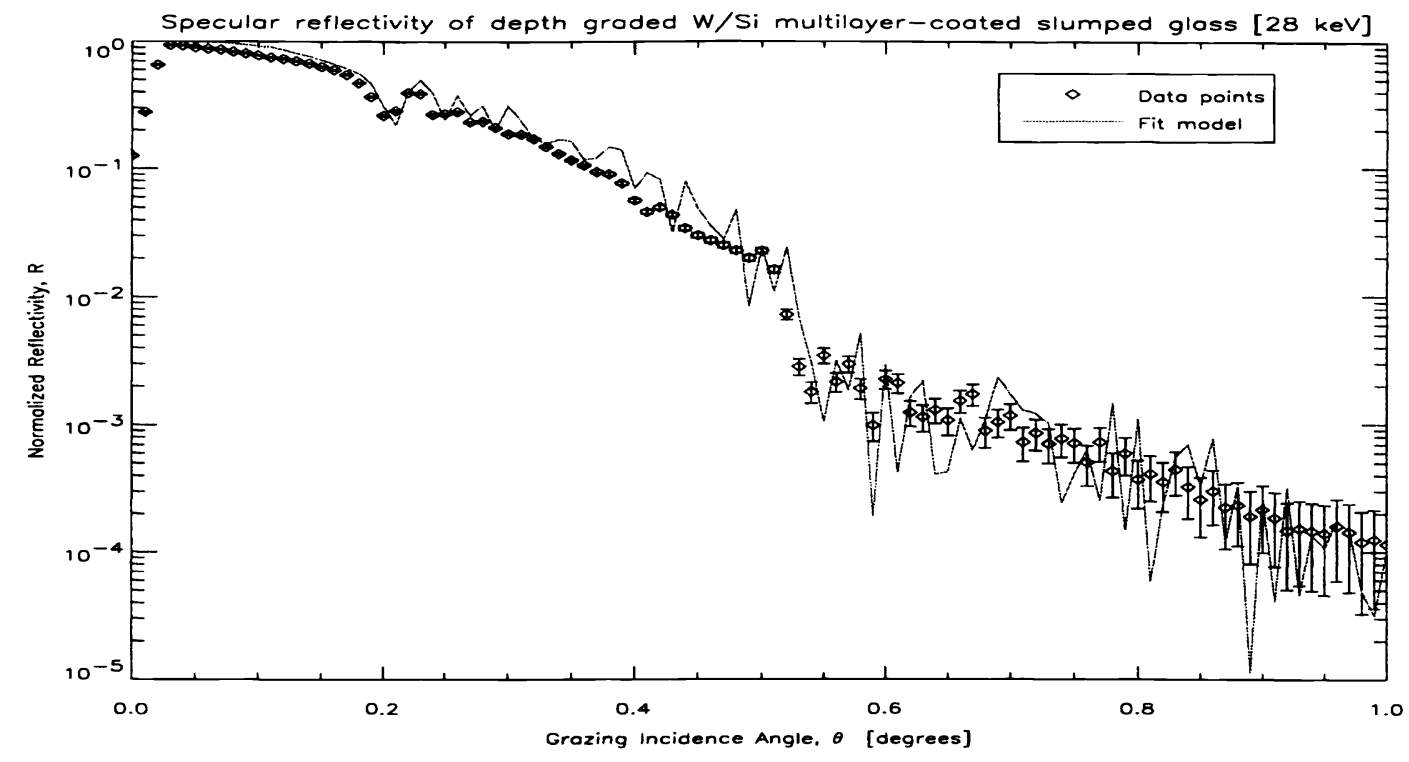

Figure 11. Specular reflectivity of W/Si depth graded multilayer-coated thermally slumped glass measured at 28 $\mathrm{keV}$. The diamonds represents the data, and the solid line is the fit (see text for details).

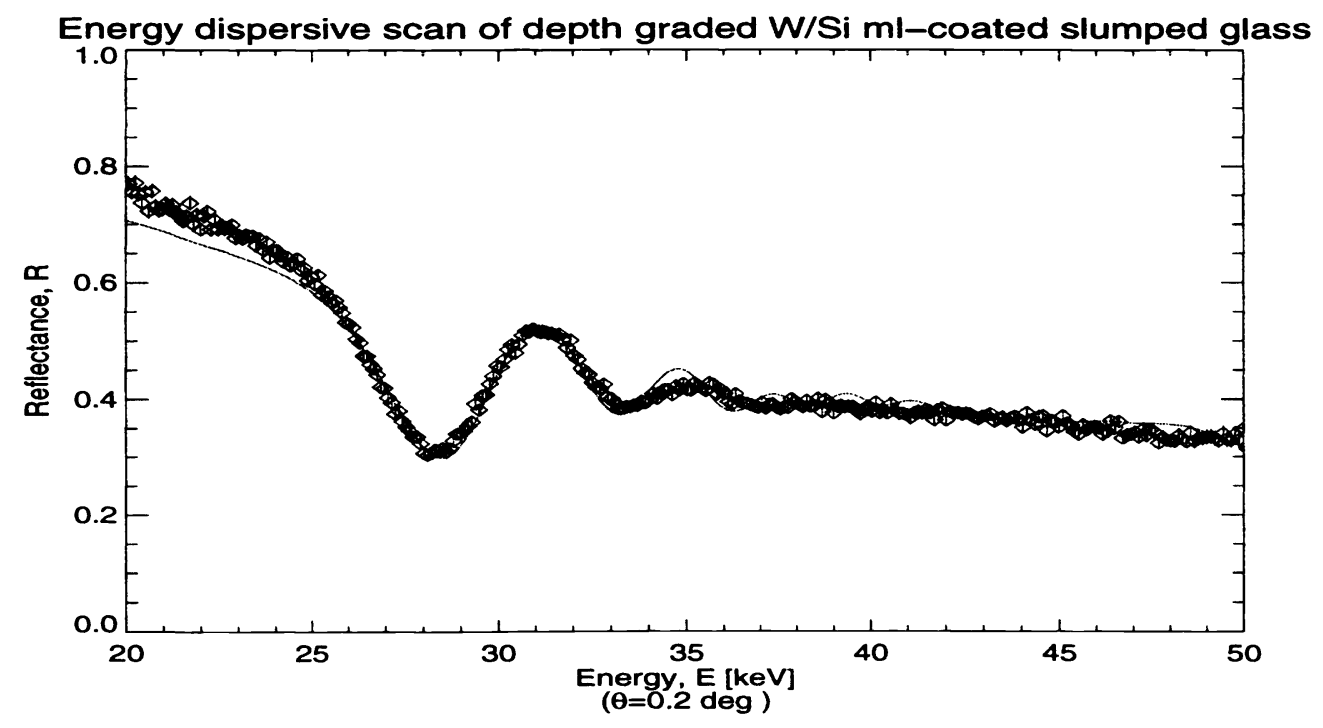

Figure 12. Normalized reflectivity as a function of energy of W/Si depth graded multilayer-coated thermally slumped glass at a grazing incidence angle of $0.2^{\circ}$. The diamonds represents the data, and the solid line is the fit (see text for details). 
the model used in the $8.048 \mathrm{keV}$ and $28 \mathrm{keV}$ specular reflectivity measurements (described in detail above). It is seen from the plot that the energy dispersive result is consistent with the $8.048 \mathrm{keV}$ and $28 \mathrm{keV}$ results, yielding an interfacial roughness of the depth graded W/Si multilayer of $\sim 4.2 \AA$.

\section{CONCLUSIONS}

We have characterized the thermally slumped glass substrates from the HEFT telescope mission using X-ray specular reflectivity and scattering measurements at $8.048 \mathrm{keV}, 18 \mathrm{keV}$ and $28 \mathrm{keV}$. We compared these results to the SODART-mirror from the $S R G$ mission.

Substantially lower r.m.s surface roughness is measured for the slumped glass compared to the SODART-mirror on the lengthscales probed in the specular reflectivity measurements. In addition, specular reflectivity and energydispersive measurements of depth graded W/Si multilayer-coated slumped glass foils yields interfacial roughness as low as $\sim 4.2 \AA$, thus demonstrating the capability of coating slumped glass substrates with high quality depth graded multilayer structures.

Scatter measurements yield Half Power Diameters (HPD's) of around 10 arcseconds for the best slumped glass substrates, and typically around 40 arcseconds for full illuminated pieces, whereas the SODART-mirror yields HPD's around 80 arcseconds. In addition, it has been shown that the HPD of the slumped glass varies with the illuminated length of the substrate, whereas the SODART-mirror maintains a constant HPD of around 80 arcseconds more or less independent of the illuminated length, indicating that the scattering from the slumped glass originates predominantly from large scale figure errors.

We tested a prototype cylindrical slumped glass fixture as well. A representative result from an un-coated mounted slumped glass showed an HPD of around 40 arcseconds, similar to the typical free-standing substrates. This shows that no increase in scatter is introduced in the mounting procedure developed for HEFT. This was also verified for the multilayer coated substrates.

We have demonstrated that with a careful selection of the thermally slumped glass substrates, the surface scatter contribution, plus any contribution from the mounting procedure, holds promise for producing a subarcminute Half Power Diameter hard X-ray telescope.

\section{ACKNOWLEDGMENTS}

The authors wish to thank the European Synchrotron Radiation Facility (ESRF) for the allocated beamtime and the technical support during the experiments, and the continuing support in this project.

\section{REFERENCES}

1. F. E. Christensen, A. Hornstrup, P. K. Frederiksen, S. Abdali, P. Grundsøe, et al., Proc. SPIE 2515 (1995), p. 458-467.

2. F. E. Christensen, A. Hornstrup, N. J. Westergaard, H. W. Schnopper, J. Wood \& K. Parker, Proc. SPIE $1546(1991)$, p. 160-167.

3. F. E. Christensen, Z. Shou-Hua, A. Hornstrup, H. W. Schnopper, P. Plag \& J. Wood, J. X-ray Sci. Tech. 3 (1991), p. 1.

4. W. W. Craig, F. E. Christensen, T. A. Decker, C. J. Hailey, F. A. Harrison, et al., Proc. SPIE 3445 (1999), p. 112-120.

5. P. Gorenstein, Hard X-ray Telescope (HXT) With Simultaneous Multiwavelength Observing From UV To 1 $\mathrm{MeV}$, Proposal for New Mission Concepts in Astrophysics, NRA 94-OSS-15, (1994).

6. F. A. Harrison et al., A Comprehensive technology Development Program for the Constellation Mission Hard $X$-ray Telescope, A Proposal to The National Aeronautics and Space Administration, (1997). 
7. F. A. Harrison (PI), The High-Energy Focusing Telescope (HEFT): A Balloon-Borne Hard X-ray/Soft GammaRay Experiment, Proposal for High Energy Astrophysics Supporting Research and Technology Program, NRA 99-OSS-01 HEA - High Energy Astrophysics, (1999).

8. F. A. Harrison \& T. A. Prince (PI's), The High-Energy Focusing Telescope (HEFT): A Balloon-Borne Hard $X$-ray/Soft Gamma-Ray Experiment, Proposal for High Energy Astrophysics Supporting Research and Technology Program, NRA 95-OSS-17, (1996).

9. A. M. Hussain, Multilayer X-ray Telescopes, Ph.D.-thesis, University of Copenhagen (1999).

10. A. M. Hussain, F. E. Christensen, G. Pareschi \& H. F. Poulsen, Proc. SPIE 3444 (1998), p. 443-450.

11. M. A. Jimenez-Garate, W. W. Craig \& C. J. Hailey, Proc. SPIE 3444 (1998), p. 622-633.

12. K. D. Joensen, F. E. Christensen, H. W. Schnopper, P. Gorenstein, J. Susini, et al., Proc. SPIE 1736 (1992), p. 239-248.

13. A. Kruse (Product Manager), Private meeting at Deutsche Spezialglas AG (DESAG) in Grünenplan, Germany (February 28. 1996).

14. P. H. Mao, F. A. Harrison, D. W. Windt \& F. E. Christensen, Appl. Optics 38(22) (1999).

15. L. Névot \& P. Croce, Rev. Phys. Appl. 15 (1980), p. 761-779.

16. L. G. Parrat, Phys. Rev. 95 (1954), p. 359-369.

17. H. W. Schnopper, Proc. SPIE 2279 (1994), p. 412.

18. H. Tananbaum, N. White \& P. Sullivan, eds., Proceedings of The High Throughput X-ray Spectroscopy Workshop, Boston, MA, 29. september - 1 October (1996).

19. D. L. Windt, Computers in Physics 12 (1998), p. 360-370.

20. D. L. Windt \& W. K. Waskiewicz, J. Vac. Sci. Technol. B 12 (1994), p. 3826-3832. 